\title{
3D modeling in the design course context: A didactic experience
}

\section{SIGRADI2018 TECHNOPOLITICAS \\ xxii congresso da sociedade iberoamericana de gráfica digital 22th conference of the iberoamerican society of digital graphics 07|08|09|novembro|2018 iau usp | são carlos | sp br}

\author{
Deborah Macêdo dos Santos \\ Universidade Federal do Cariri, IISCA | Brazil | deborah.santos@ufca.edu.br
}

\begin{abstract}
The use of informatic tools (computer-aided design) may be associated to designer tasks since the first phase of product conception until industrial production. By using these CAD tools during the initial design phase, it is possible to predict, identify and correct mistakes. This is an interdisciplinary article that presents and discusses an experience as a teacher of graphics computation II, offered to product design students. It also reveals the didactic methods and psychological approaches applied to address this challenge: Achievement motivation, pedagogy of autonomy and learning pyramid. The didactic experiment was positive and lead to interesting learning results.
\end{abstract}

Keywords: 3D modeling; CAD; Product design; Teaching; Learning.

\section{INTRODUÇÃO}

Do pacote de métodos apropriados para o ensino de design de produtos, o uso de ferramentas informáticas do tipo de desenho auxiliado por computador CAD (computer aided design) tem sido amplamente aceito desde os anos 80 (Green \& Bonollo, 2002). Desde então, como consequência, o processo do projeto se tornou mais preciso e aplicado conceitos de maior qualidade.

Seu uso pode estar associado às tarefas do designer desde a primeira fase de concepção até a produção industrial dos produtos. Na fase de concepção, estes programas permitem estudos estéticos volumétricos, com aproximação prévia do produto final e apresentação inclusive de cores reais dos materiais utilizados (Díaz, 2016; Santos, Araújo, Oliveira, \& Costa, 2015). Admitem uma experimentação mais ousada em relação a sua liberdade formal, facilitando a criação de projetos inovadores (Ferreira Neto \& Santos, 2015; Muñoz, Coronel, Magnasco, \& Sequeira, 2012). É possível ainda prever, identificar e corrigir erros na fase inicial de criação com estas ferramentas.

Na fase de produção, em muitos casos, é mais prático e rápido a indústria criar protótipos, ou mesmo fôrmas para protótipos ou produtos finais, por meio da impressão 3D de peças vindas das ferramentas CAD. Quando aplicadas no desenvolvimento de produtos, os projetos podem apresentar resultados positivos diretos na qualidade, precisão e agilidade das novas peças (Celani, 2003; Maciel, Amorim, \& Checcucci, 2018), ainda na diminuição do desperdício de materiais oriundos de protótipos físicos sem estudo prévio.

Como transmitir esse conhecimento técnico aos novos designers promovendo uma autonomia intelectual? Este artigo apresenta e discute a temática interdisciplinar das práticas pedagógicas, aplicadas no ensino da modelagem geométrica. Trata-se de uma aplicação prática, da experiência vivenciada na disciplina Computação Gráfica
II, ofertada aos alunos do curso tecnólogo em Design de Produto numa Universidade Pública Federal, na região do semiárido cearense, no ano letivo de 2015.1.

O ensino de ferramentas CAD nos cursos de design de produtos tem sido estimulado, nas instituições mais tradicionais. Contudo, mesmo com tanta prática profissional, ainda são poucas as publicações que apresentam os resultados didáticos e discutem sua prática pedagógica. Este artigo objetiva apresentar e discutir, de maneira interdisciplinar, a luz dos estudos de educação, as estratégias pedagógicas para o ensino da modelagem geométrica, com o uso do software Rhinoceros, no curso de design de produtos.

\section{CONTEXTO CIENTíFICO E MOTIVAÇÃO}

Ensinar exige constante reflexão crítica sobre a prática docente (Freire, 2011). A busca por métodos e estratégias de ensino que possam tornar o processo de aprendizagem mais estimulante, deveria ser uma preocupação de todos professores, que permeia todas as áreas do saber (Ponte, Miranda, \& Santos, 2016). Esta lógica parece não se encaixar quando se busca por publicações que apresentam o ensino de ferramentas de CAD no curso de design de produtos. Mais escasso ainda é encontrar nesta temática, uma associação às suas estratégias pedagógicas.

Em contraponto, os estudiosos das práticas pedagógicas no ensino básico têm devotado esforço em publicações sobre este tema. Vastas são as publicações e eventos dedicados ao ensino das novas tecnologias com olhar no ensino básico. Como por exemplo: o uso das TICs (Tecnologia da Informação e Comunicação) em sala de aula, o ensino de informática, programação e fabricação digital para crianças, entre outros. Quando direcionadas ao ensino superior, sua maioria é dedicada aos profissionais de informática ou engenharias. Algumas das referências pedagógicas utilizadas como apoio de embasamento teórico ao longo deste artigo vem destas fontes. 
Para confirmar esta carência de referências no tema, foi feita uma pesquisa prévia no buscador científico Web of Science, classificado como uma ampla e credível fonte de publicações acadêmicas (Silva Neto, Cruz, Rodrigues, \& Silva, 2016). Os descritores utilizados foram $C A D$ e TEACH. Foram encontrados 722 resultados. A categoria que inclui os estudos de design é a intitulada como arquitetura. Esta aparece em décimo nono lugar com vinte artigos, sendo quatro oriundos de revistas científicas e dezesseis de conferências. Dos vinte artigos, apenas quatro tem aplicação nomeada ao designer. Os outros ou são aos estudantes de arquitetura ou tem aplicação genérica.

Dos quatro artigos que contém as palavras CAD e ensino (em inglês), e que contemplam o estudante de design, todos são de conferências. São estas: Advances in architectural geometry, Caadria e eCAADe (este último com dois artigos).

O primeiro artigo analisado define e descreve maneiras de modelar sulcos de papel dobrado, uma das conclusões é que a aplicação do método facilitaria o ensino mais preciso para designer (Koschitz, 2016).

O segundo discute estratégias de ensino do fluxo entre CAD/CAM para estudantes iniciantes de arquitetura e design. Fica evidente a importância de se perceber, no início do curso, a diferença entre limites do objeto virtual e real (Gannon \& Brockmeyer, 2014).

O terceiro apresenta uma biblioteca para desenho paramétrico por meio de programação em linguagem JAVA. Acreditava-se que tanto a pesquisa como o ensino precisariam dessa ferramenta para ultrapassar as limitações dos programas de desenho de produtos (Labelle, Nembrini, \& Huang, 2010).

O quarto artigo analisado afirma que as ferramentas que permitem ao designer trabalhar com formas, padrões e modelos não garantem que o conhecimento nem o ensino seja adequado a técnica apropriada. Deste modo o artigo apresenta desenhos feitos com auxílio do computador (Gün, 2009).

Ou seja, por meio da base de dados consultada, não foram encontradas discussões com ênfase na prática pedagógica para o ensino de CAD. Esta pesquisa é motivada pela carência de publicações relacionadas ao tema e pretende ser um contributo para a busca da formação de indivíduos com autonomia intelectual, e ainda eventuais estudos sobre o desenvolvimento de uma melhor relação de ensino e aprendizagem nesta matéria.

\section{DESENVOLVIMENTO, PEDAGOGIA E DISCUSSÕES}

O método empregado para vencer este desafio, baseou-se na estratégia "face a face" com aulas presenciais (Unver, 2006), na redução de aulas exclusivamente expositivas, priorizando a realização de vários exercícios de modelagem e renderização, que abordasse prioritariamente as temáticas de calçados e joias, que são ênfase do curso.
A amostra era intencional, formada por um grupo heterogêneo de inicialmente 29 pessoas, todos estudantes regulares do curso de design de produtos. Houve 7 desistências ainda no início do curso por motivos particulares diversos como por exemplo choque de horário com empregos e uma gestante que precisou de repouso. Dos 22 que concluíram, nenhum possuía experiência anterior com algum software de modelagem.

A estratégia pedagógica explorada foi a pedagogia da autonomia. Isto por meio da abordagem psicológica que une a motivação de realização e a pirâmide de aprendizado. Estas estratégias e abordagens ocorreram de maneira conjunta, complementando-se umas às outras em cada atividade. Entretanto foram separadas na escrita deste artigo, de acordo com as atividades que possuem maior percepção de sua ação, para melhor explanação.

\section{MOTIVAÇÃO DE REALIZAÇÃO}

São os estudantes mais motivados que tiram melhor proveito dos recursos que lhes são dispostos (Miranda, 2007). Embora se reconheça que os fatores sociais, origens e experiências individuais de cada aluno podem influenciar nas motivações de maneiras diferentes, também é possível criar estratégias para manter os alunos motivados, neste caso procurou-se aplicar a teoria de motivação por realização (Pontes \& Miranda, 2018; Revelle \& Michaels, 1976).

A teoria da motivação por realização foi criada por John Atkinson e David McClelland (Atkinson \& Feather, 1966). Esta teoria define a realização humana pelo o resultado da relação antagônica entre o esforçar-se pelo sucesso e o evitar falhar (Covington, 1998). Essas duas ações podem ser condutoras da motivação, uma desenvolvendo estratégias que objetivam o sucesso, a outra procurando estratégias que evitam o falhanço.

No esforçar-se pelo sucesso, por exemplo, pode-se encorajar a mentalizar o sucesso e a sensação do orgulho antecipadamente para motivar pessoas a alcançar a excelência. Por outro lado, ao mentalizar-se antecipadamente numa situação de vergonha e humilhação, faz com que pessoas possam evitar situações em que elas acreditam que vão falhar. Esta ação, de permitir-se experimentar antecipadamente o orgulho ou a vergonha, pode ajudar a encontrar o porquê de algumas pessoas aceitarem o aprendizado com entusiasmo, enquanto outras o fazem com relutância (Covington, 1998).

Um aluno desmotivado pode não ter sucesso em seus estudos e até abandonar o curso. Pesquisadores acreditam que a motivação deve ser valorizada no contexto escolar. Este artigo defende que também deve ser valorizada no contexto universitário, pelos mesmos motivos: a motivação produz uma maior satisfação na realização dos trabalhos, proporciona melhor aprendizagem, estimula autonomia e confiança dos alunos em si próprios (Lemos, 2010)

É sabido que os alunos mais motivados, possuem maior resiliência frente aos obstáculos Os estudantes mais inclinados a 'evitar falhar', apresentam baixa resistência 


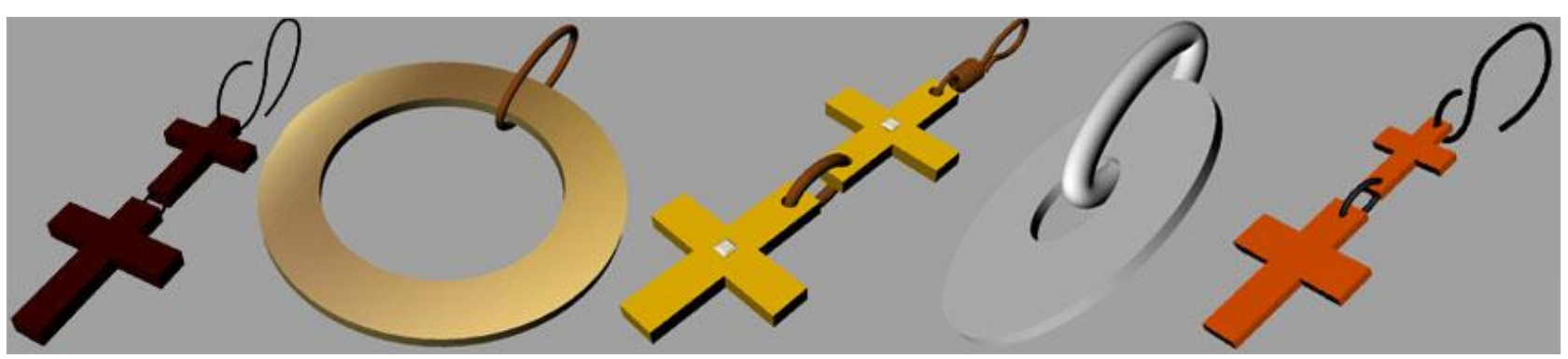

Figura 1: trabalhos aleatórios da primeira aula de discentes com a temática joia. Modelagem e renderização com Rhinoceros. Fonte: Autora.

aos obstáculos, se frustram, aprendem menos, desistem mais, e são inclinados a escolher tarefas ou muito fáceis ou muito exigentes para suas capacidades, pois avaliam pior as situações que Ihe são propostas (Lieury \& Fenouillet, 1997).

Com base nesta estratégia foi montada uma primeira aula teórica com um único objetivo: Motivar. Foram apresentadas diversas ilustrações de renders de aplicações práticas do uso da ferramenta em escalas diferentes. Modelos de joias, calçados, moda, casas, cidades. Foi apresentado ainda a facilidade para criar protótipos com o uso de impressoras 3D, máquinas injetoras, entre outros, depois que o modelo tridimensional está feito. $\mathrm{O}$ aluno passou a perceber melhor o motivo da existência daquela disciplina (Computação gráfica), o seu contexto no curso de design e como poderão aplicar os conhecimentos da disciplina no futuro profissional.

$\mathrm{Na}$ sequência, houve a primeira aula prática. Talvez a maneira mais tradicional de se ensinar a manipular um software seja apresentar a interface e mostrar as ferramentas pela ordem em que elas aparecem. Como estratégia de motivação foi decidido, logo após uma apresentação rápida da interface, dar aos alunos uma perspectiva de tarefa realizada: "hoje, todos irão modelar pelo menos uma joia". Os relatos dos aprendizes a partir deste desafio diário era de que: uns diziam ser impossível, outros se riam, outros apenas aceitaram o desafio e outros ainda ficaram entusiasmados.

Foi explicada uma modelagem prática semelhante a que eles teriam de fazer, e as ferramentas do software CAD em estudo iam sendo apresentadas conforme a necessidade do modelo, os aprendizes acompanhavam de maneira individual. Com a rotina de Ihes apresentar pontualmente as ferramentas que necessitavam para a realização daquela prática, compreendeu-se que naquele momento os alunos adquiriam o conhecimento, mesmo que inicial do funcionamento delas.

Depois da seção expositiva, foi Ihes fornecido um guia ilustrado de outro exercício que utilizava as mesmas ferramentas. Aqui com a ideia de mensurar a transferência do conhecimento logo adquirido numa nova atividade.

Ao final da primeira aula todos concluíram a modelagem e renderização simples de duas joias, um pingente circular e um brinco pendente com crucifixos (tamanho, quantidade e cores variaram segundo o gosto de cada um). Os alunos apresentaram seus modelos visivelmente orgulhosos. A Figura 1 é um exemplo de trabalho desenvolvido no primeiro dia de aula prática.

\section{PEDAGOGIA DA AUTONOMIA}

Para que a estratégia não se bastasse no transferir de conhecimento, era necessário ainda criar a possibilidade para sua própria produção como estratégia de desenvolver a autonomia do aprendizado (Freire, 2011). Isto é o que defende a pedagogia da autonomia, desenvolvida pelo autor Paulo Freire.

Esta pedagogia se propõe a cultivar e desenvolver o conhecimento em sala de aula a partir as experiências de todos. Neste caso o professor não é o detentor de todo o conhecimento a ser transferido, mas um facilitador da promoção do conhecimento (Freire, 2011).

Neste caso de estudo, a pedagogia da autonomia foi aplicada durante todo o semestre letivo. Era solicitado a cada aula, que as peças fossem personalizadas, coloridas, adornadas de acordo com o gosto de cada um. A Figura 2 exemplifica isto com alguns modelos aleatórios de calçados, desenvolvido pelos alunos, na décima aula prática.

Paulo Freire diz que a autonomia do aluno se funda na responsabilidade que lhe vai sendo confiada. Neste caso, estes alunos tinham de, a cada aula de exercício prático, entregar um modelo.

As temáticas aplicadas aos exercícios eram modelagens de calçados e joias (ênfase do curso tecnólogo), ainda outras temáticas, como modelagem mobiliário, mandala e personagens de animação digital, também foram estimuladas a fim de favorecer o desenvolvimento da pesquisa de maneira interdisciplinar (Fontoura, 2011)

A pedagogia da autonomia destaca ainda a importância de criar situações de socialização entre os alunos (Freire, 2011). Pesquisas com esta abordagem apontam que os alunos devem se assumir como seres pensantes, para tal as aulas devem ser centradas na aprendizagem colaborativa (Pontes, 2013).

Neste estudo, em cada aula, os alunos que apresentavam maior facilidade de aprendizagem eram solicitados a ajudar seus pares. A depender do exercício, alguns tinham mais facilidade que outros, então a dinâmica de ajudar e ser ajudado, não necessariamente se tornava repetitiva para as mesmas pessoas.

Por meio de exercícios temáticos - em sua maioria - era percebida a assimilação dos alunos das possibilidades de uso do software com as atividades práticas. 


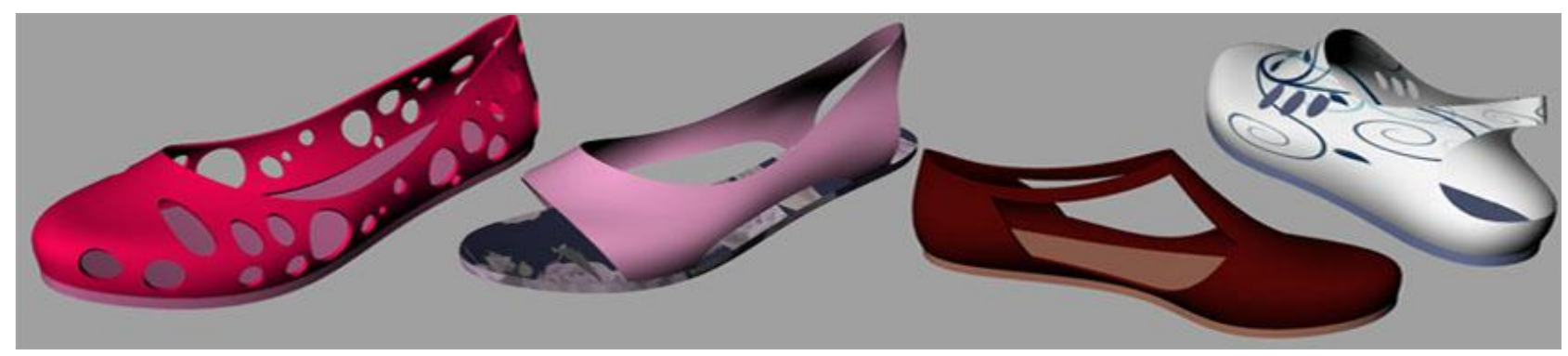

Figura 2: trabalhos aleatórios de discentes com a temática calçado. Modelagem e renderização com Rhinoceros. Fonte: Autora.

Com a resolução dos exercícios na exploração das funções nativas do software, os alunos iam adquirindo, cada um em seu tempo, novas habilidades e competências. Ao fim de cada atividade prática, os trabalhos eram apresentados para a sala, assim podiam ser avaliados e discutidos por todos.

Estas exposições por parte dos alunos tinham ainda o objetivo de perceber o nível de aprendizagem de cada um, para então caso necessário buscar estratégias de nivelamento da turma no mesmo patamar, de acordo com o plano de aulas da disciplina, buscando garantir assim o sucesso escolar ao fim do tempo da disciplina.

\section{PIRÂMIDE DE APRENDIZADO}

Não se sabe a origem exata da pirâmide aprendizado que conhecemos hoje, mas muitas publicações à referem como associação dos trabalhos do educador Edgard Dale e do psicólogo William Glasser (Molenda, 2003; Ribeiro et al., 2018; Roman et al., 2017). Embora sua origem dos estudos empíricos seja desconhecida (Letrud \& Hernes, 2016), é fato que muitos experimentos didáticos têm sido bem sucedidos com a aplicação da mesma. Este é mais um deles.

Este modelo demonstra o quanto o cérebro humano retém de informação após cada atividade (Dale, 1969; Glasser, 1986). As proporções do modelo são:

- $10 \%$ - Ler

- $20 \%$ - Ouvir

- $30 \%$ - Ver

- $50 \%$ - Ver e ouvir

- $70 \%$ - Interagir

- $80 \%$ - Praticar

- $95 \%$ - Ensinar aos outros

No experimento desta pesquisa, foram feitas diversas apresentações orais com o objetivo de que os alunos pudessem se aproximar, ao máximo, da base da pirâmide de aprendizado. Resultando numa maior absorção e produção dos conhecimentos. A avaliação de cada nova atividade era com apresentação coletiva dos resultados com discussões abertas. Os alunos apresentavam seu modelo, o conceito, que ferramentas utilizaram, onde tiveram maior dificuldade, entre outros. Estas apresentações, abertas a intervenções da turma, também promoviam um espaço onde o conhecimento podia ser discutido e desenvolvido.

\section{AUTONOMIA NA AVALIAÇÃO FINAL}

A questão proposta para a avaliação final, seria que todos apresentassem uma peça autoral modelada com o software Rhinoceros (Exemplos aleatórios apresentados nas figuras 3 e 4 ).

Cada aluno traz um conhecimento intrínseco. A pedagogia da autonomia defende que este conhecimento deve ser respeitado e ainda aproveitado em sala de aula (Freire, 2011). Por isso o tema do modelo foi livre. Os alunos podiam tomar como base sua experiência particular, na área que mais Ihes apetecesse, para criar seu modelo e conceitua-lo.

Deixar o tema livre poderia ainda Ihes conectar com outras disciplinas, já que muitos cursavam, em paralelo, aulas de projeto. Desta maneira, este trabalho final poderia servir de representação visual complementar para outras atividades, caso assim desejassem.

Ao aplicar o modelo em outras disciplinas, ou ao conectar com os conhecimentos múltiplos de cada aluno, o objetivo também é ajudar na promoção da interdisciplinaridade. Isto é importante para lhes ajudar a vencer, na carreira profissional, os desafios modernos, que exigem soluções complexas e interdisciplinares (Bursztyn, 2004), no contexto de uma profissão que por si só já nasce interdisciplinar (Beirão, 2017; Fontoura, 2011).

Por meio de experimentos, pesquisadores descobriram que os alunos que tem mais sucesso com na resolução dos problemas, tendiam a estudar, escrevendo um texto a parte com explicações e justificativas para cada ação (Chi, Bassok, Lewis, Reimann, \& Glaser, 1989). Assim sendo, com a intenção de aumentar as condições de sucesso dos alunos nesta tarefa final, foi lhes solicitado também o desenvolvimento de um guia ilustrado explicando os pormenores de como modelar a peça.

Aprender exige do aluno um processo de conversão dos exemplos e palavras fornecidos pelo professor em habilidades práticas (Chi et al., 1989). Este processo é uma das maneiras de aprendizado com autonomia.

Como atividade final, foi pedida a apresentação de um projeto original de tema livre em moldes de tutorial. Os resultados foram categorizados posteriormente nos subtemas: calçados, joias, mobiliário e elementos de animação. Estes foram considerados significativos para aferir a percepção da qualidade final do aprendizado, desenvolvimento da criatividade e autonomia dos alunos diante de novos problemas, ainda, serviram de material para auxiliar na formação das próximas turmas.

Ao final do semestre, antes de receberem as notas, os alunos foram convidados a espontaneamente, de maneira anônima, responderem a um questionário institucional de 


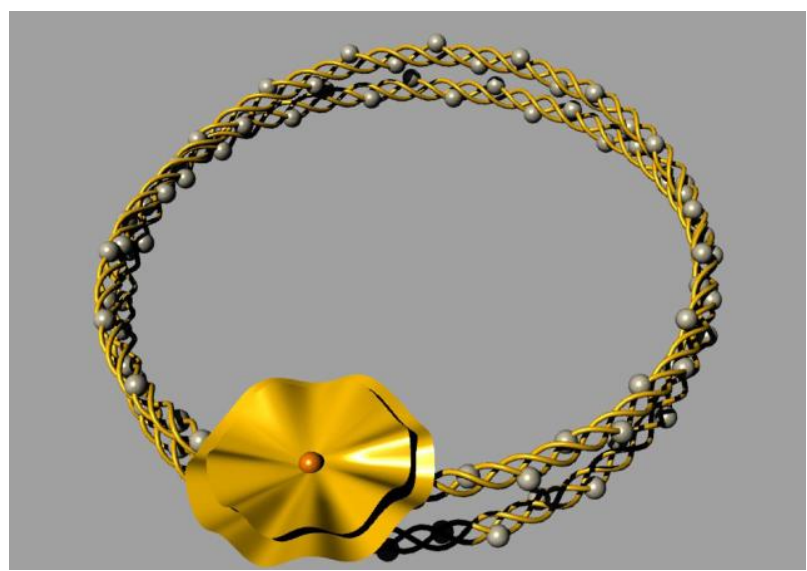

Figura 3: Gargantilha modelada por discente para apresentação final. Fonte: Autora.

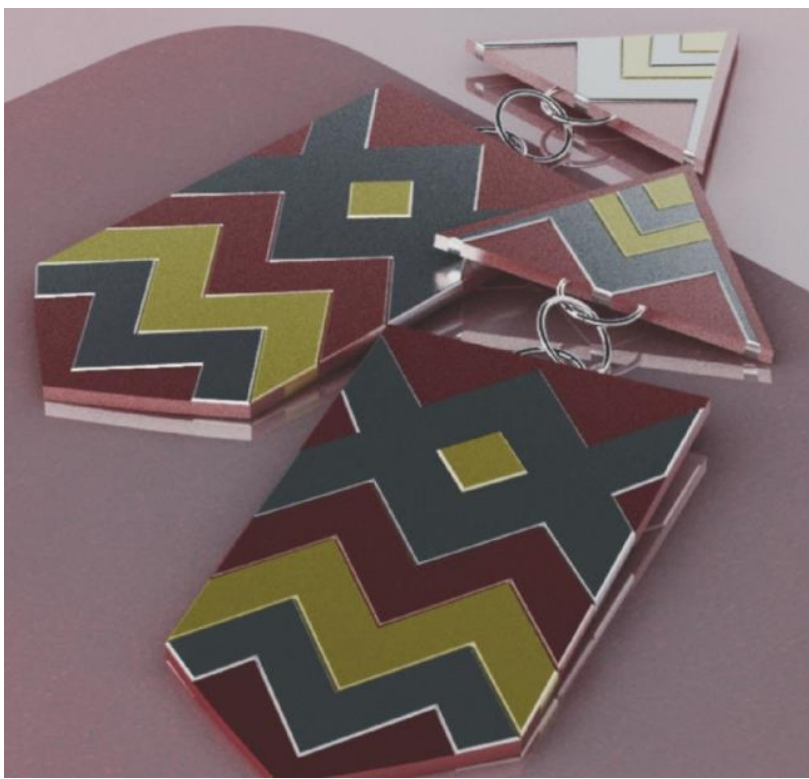

Figura 4: Brincos modelados por discente para apresentação final. Fonte: Autora.

avaliação didática do professor. Quinze alunos decidiram participar. Esta avaliação abordava quatro dimensões que seriam respondidas numa escala de 1 a 5, são elas: Planejamento pedagógico, didático e domínio do conteúdo; Relacionamento e postura com os discentes; Formas e usos da avaliação do aprendizado discente; Pontualidade e assiduidade as aulas. Os resultados estão sumarizados na Tabela 1.

Tabela 1: Avaliação pedagógica feita por alunos

\begin{tabular}{ll}
\hline Dimensões & Total $(0-5)$ \\
\hline D1 & 4,68 \\
D2 & 4,76 \\
D3 & 4,74 \\
D4 & 4,97 \\
\hline MD & 4,77
\end{tabular}

\section{RESULTADOS E DISCUSSÕES}

A motivação de realização no processo de aprendizagem se mostrou um fator positivo, por vezes, os alunos motivados faziam, espontaneamente, mais do que o solicitado nas aulas e atividades. Isto demonstrou, por parte deles, um comportamento ativo no processo de aprendizagem e como consequência, a turma apresentou bom domínio das ferramentas estudadas ao final da disciplina.

Em toda aula prática pedia-se que depositassem, na nuvem coletiva, um modelo temático customizado pelo aluno. Foi recorrente que alunos, espontaneamente, enviassem dois modelos diferentes. Quando questionados, as justificativas eram: "tive novas ideias durante a modelagem do primeiro, ainda dava tempo de fazer outro e não consegui decidir qual ficou melhor".

As atividades da pirâmide de aprendizado, foram todas exploradas em sala, bem como a pedagogia da autonomia. Os alunos com mais facilidade aceitaram o convite de ajudar os que demoravam mais para assimilar as ferramentas. Sob minha supervisão eram instruídos a não "fazer por eles", mas ajudar no caminho lógico para que eles descobrissem por si. O professor, de maneira prática, deixou de ser o único detentor do conhecimento, passando a ser um facilitador.

Dos 29 alunos, 22 foram aprovados na disciplina. 7 desistiram ainda no começo por motivos pessoais. A pessoa com mais dificuldade durante o semestre, declarou não possuir computador em casa, mas ainda conseguiu classificação razoável devido a carga de exercícios executados na sala.

Os momentos de partilha de conhecimento (apresentações orais) ao fim de cada aula foram enriquecedores para o desenvolvimento do conhecimento em sala, para a autonomia e motivação dos futuros designers.

O trabalho final de tema livre, promoveu a interdisciplinaridade. Alguns alunos fizeram modelos que serviram e complemento para outras disciplinas, outros, de maneira espontânea, trouxeram temáticas de diferentes culturas e áreas do saber para conceituar seus modelos. Além do ficheiro do modelo e do documento escrito de como foi desenvolvido, todos foram apresentados e discutidos em sala.

Por fim, outra importante realização deste artigo, foi mostrar o potencial do uso de ferramentas computacionais no processo criativo. Ficou evidente a diversidade de soluções apresentada pelos alunos, a cada exercício, que tinham a restrição de utilizar as mesmas ferramentas que os demais. Contradizendo o mito de que o uso das ferramentas computacionais inibe a criatividade dos alunos e leva a soluções padronizadas.

\section{AGRADECIMENTOS}

Agradecemos aos alunos de design da Universidade Federal do Cariri por inspiradora dedicação; Ao CNPq (Conselho Nacional de Desenvolvimento Científico e Tecnológico) pela manutenção da bolsa de estudos (201904/2015-2); Ao CIAUD (Centro de investigação em arquitetura, urbanismo e design) da Universidade de Lisboa pelo apoio. 


\section{REFERÊNCIAS}

Atkinson, J. W., \& Feather, N. T. (Eds.). (1966). A Theory of Achievement Motivation Title: A Theory of Achievement Motivation (66th ed.). New York: Wiley.

Beirão, J. N. (2017). Sobre o ensino da Arquitectura e o futuro profissional do Arquitecto $\mathrm{O}$ papel da Arquitectura nas sociedades criativas. Retrieved June 15, 2018, from http://www.jornalarquitectos.pt/pt/forum/cronicas/sobre-oensino-da-arquitetura-e-o-futuro-profissional-do-arquiteto

Bursztyn, M. (2004). Meio ambiente e interdisciplinaridade: desafios ao mundo acadêmico. Desenvolvimento e Meio Ambiente, (10) https://doi.org/http://dx.doi.org/10.5380/dma.v10i0.3095

Celani, G. (2003). CAD Criativo. Rio de Janeiro: Campus.

Chi, M. T. H., Bassok, M., Lewis, M. W., Reimann, P., \& Glaser, R. (1989). Self-explanations: How students study and use examples in learning to solve problems. Cognitive Science, 13(2), 145-182. https://doi.org/10.1016/0364-0213(89)900025

Covington, M. V. (1998). The will to learn: a guide for motivating young people. Cambridge University Press. Retrieved from https://books.google.com.br/books?hl=pt$B R \& \mid r=\& i d=j 4 V Z v W F-X d 8 C \& o i=f n d \& p g=P A 1 \& d q=$ Covington+1998\&ots=0amW6hwdlw\&sig=zcZLrhqnAkaZvR1 J7jZInCJ_HIU\#v=onepage\&q=Covington 1998\&f=false

Dale, E. (1969). Audiovisual methods in teaching. NY: Dryden Press. https://doi.org/10.1108/00400910910987273

Díaz, D. T. (2016). Tecnologías de Fabricación Digital Aditiva, ventajas para la construcción de modelos, prototipos y series cortas en el proceso de diseño de productos. Iconofacto, 12(January), https://doi.org/10.18566/iconofac.v12n18.a07

Ferreira Neto, F. L., \& Santos, D. M. (2015). A modelagem digital 3D como ferramenta no design de joias. In C. T. F. Costa, R Colares, D. A. R. Mota, \& H. P. C Oliveira (Eds.), Cadernos de experiências: Pesquisa em foco (pp. 179-184). Juazeiro do Norte: UFCA. Retrieved from https://ebooks.ufca.edu.br/catalogo/pesquisa-em-foco-v1/

Fontoura, A. M. (2011). A Interdisciplinaridade e o ensino do design. Projética Revista Científica de Design, 2(2), 86/95.

Freire, P. (2011). Pedagogia da autonomia: saberes necessários à prática educativa. São Paulo: Editora Paz e Terra. https://doi.org/10.4324/9780203420263

Gannon, M., \& Brockmeyer, E. (2014). Teaching CAD/CAM workflows to nascent designers. In N. Gu, S. Watanabe, H. Erhan, M. H. Haeusler, W. Huang, \& H. Kong (Eds.), Caadria (pp. 811-820).

Glasser, W. (1986). Control theory in the classroom. Control theory in the classroom

Green, L. N., \& Bonollo, E. (2002). The Development of a Suite of Design Methods Appropriate for Teaching Product Design. Global Journal of Engineering Education, 6(1), 45-53.

Gün, O. Y. (2009). Value through Precision, Beyond the Realms. In 27th eCAADe Conference Proceedings (pp. 377-384). Istanbul.

Koschitz, D. (2016). Designing with Curved Creases Digital and Analog Constraints. In Advances in architectural geometry (pp. 82-103). Zurich.

Labelle, G., Nembrini, J., \& Huang, J. (2010). ANAR + : Geometry library for Processing. In G. Schmitt, L. Hovestad, M. VanGool L, F. Bosche, R. Burkhard, S. Coleman, ... M. SehmiLuck (Eds.), eCAADe (pp. 403-410). Zurique.

Lemos, M. S. (2010). Motivação e aprendizagem. In G. L. Miranda \& S. Bahia (Eds.), Psicologia da educação: temas de desenvolvimento, aprendizagem e ensino (2nd ed., p. 368). Relógio D’Água Editores.
Letrud, K., \& Hernes, S. (2016). The diffusion of the learning pyramid myths in academia: an exploratory study. Journal of Curriculum Studies, 48(3), 291-302. https://doi.org/10.1080/00220272.2015.1088063

Lieury, A., \& Fenouillet, F. (1997). Motivação e Sucesso Escolar (1st ed.). Lisboa: Editorial presença.

Maciel, S. D., Amorim, A. L. de, \& Checcucci, É. D. S. (2018). Ensino De Projeto De Arquitetura Em Ambiente Digital: Uma Experiência Na Faculdade De Arquitetura Da Universidade Federal Da Bahia. Gestão \& Tecnologia de Projetos, 13(1), 21. https://doi.org/10.11606/gtp.v13i1.133839

Miranda, G. L. (2007). Limites e possibilidades das TIC na educação. Sísifo. Revista de Ciências Da Educação, 3(2007), 41-50. https://doi.org/10.1590/S0100-19651997000200006

Molenda, M. (2003). Cone of Experience. In Educational Technology: An Encyclopedia (pp. 161-165). Santa Barbara, CA: ABC-Clio.

Muñoz, P., Coronel, J. L., Magnasco, I. R., \& Sequeira, A. (2012). La flexibilidad en la generación de formas. (P. Muñoz, Ed.). Buenos Aires: Ediciones de la forma.

Ponte, T. B., Miranda, G. L., \& Santos, D. M. dos. (2016). A programação de computadores para alunos de arquitectura: Uma análise do uso da linguagem Racket para protótipos 3D. In N. Pedro, A. Pedro, J. F. Matos, J. Piedade, \& M. Fonte (Eds.), Digital Technologies \& Future School. Atas do IV Congresso Internacional das TIC na Educação (artigos selecionados) (pp. 197-208). Lisbon: Instituto de Educação da Universidade de Lisboa. Retrieved from https://cld.pt/dl/download/e7500488-3c2a-4d99-9de0ade4c5cc9aba/Livro Artigos.pdf

Pontes, T. B. (2013, November 8). Planejamento de aula no ensino superior na modalidade blended learning: requisitos para rede social educativa. Universidade Federal de Pernambuco. Retrieved from https://repositorio.ufpe.br/handle/123456789/12435

Pontes, T. B., \& Miranda, G. L. (2018). Validação em Língua Portuguesa da Escala de Motivação de Realização. Risti, (27), 109-125. https://doi.org/10.17013/risti.27.109

Revelle, W., \& Michaels, E. J. (1976). The theory of achievement motivation revisited: The implications of inertial tendencies. Psychological Review, 83(5), 394-404. https://doi.org/10.1037/0033-295X.83.5.394

Ribeiro, A. L., Fonseca, L. A. N. S., Elias, T. A. S., Silva, A. R. O. S., Santos, R. A. dos, \& Silva, A. M. da. (2018). Promoção do ensino de programação e robótica a estudantes de escolas da rede pública de ensino na cidade de Divinópolis. In Anais do Computer on the Beach (Vol. 0, pp. 631-640). Retrieved from https://siaiap32.univali.br/seer/index.php/acotb/article/view/12 934/7403

Roman, C., Ellwanger, J., Becker, G. C., Silveira, A. D. Da, Machado, C. L. B., \& Manfroi, W. C. (2017). Metodologias ativas de ensino-aprendizagem no processo de ensino em saúde no Brasil: Uma revisão narrativa. Clinical \& Biomedical Research, 37(4), 349-357. https://doi.org/10.4322/23579730.73911

Santos, D. M., Araújo, M. D. X., Oliveira, H. P. C., \& Costa, C. T. F. (2015). Modelagem tridimensional com Rhinoceros: Guias llustrados. Juazeiro do Norte: UFCA. Retrieved from https://ebooks.ufca.edu.br/catalogo/modelagem-comrhinoceros/

Silva Neto, D. de C. e, Cruz, C. O., Rodrigues, F., \& Silva, P. (2016). Bibliometric Analysis of PPP and PFI Literature: Overview of 25 Years of Research. Journal of Construction Engineering and Management, 142(10), 06016002. https://doi.org/10.1061/(ASCE)CO.1943-7862.0001163

Unver, E. (2006). Strategies for the Transition to CAD Based 3D Design Education. Computer-Aided Design and Applications, 3(1-4), 323-330. 